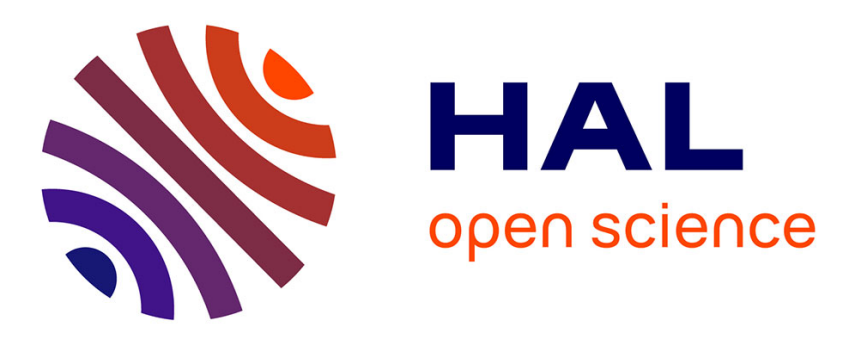

\title{
Real time 3D US-tagging combined with 3D phase-based motion estimation
}

\author{
Sébastien Salles, H. Liebgott, Damien Garcia, D. Vray
}

\section{To cite this version:}

Sébastien Salles, H. Liebgott, Damien Garcia, D. Vray. Real time 3D US-tagging combined with 3D phase-based motion estimation. 2013 IEEE International Ultrasonics Symposium (IUS), Jul 2013, Prague, Czech Republic. pp.585-588, 10.1109/ULTSYM.2013.0151 . hal-00850061

\section{HAL Id: hal-00850061 https://hal.science/hal-00850061}

Submitted on 22 Jul 2019

HAL is a multi-disciplinary open access archive for the deposit and dissemination of scientific research documents, whether they are published or not. The documents may come from teaching and research institutions in France or abroad, or from public or private research centers.
L'archive ouverte pluridisciplinaire HAL, est destinée au dépôt et à la diffusion de documents scientifiques de niveau recherche, publiés ou non, émanant des établissements d'enseignement et de recherche français ou étrangers, des laboratoires publics ou privés. 


\title{
Real time 3D US-tagging combined with 3D phase- based motion estimation.
}

\author{
Sebastien Salles ${ }^{1}$, Hervé Liebgott ${ }^{1}$, Damien Garcia ${ }^{2}$, Didier Vray ${ }^{1}$ \\ 'CREATIS, CNRS UMR 5220, INSERM U1044, INSA-lyon, Université de Lyon, France, \\ ${ }^{2}$ CRCHUM, University of Montreal, Departement of radiology, Canada
}

\begin{abstract}
By contrast with 2D imaging, quantitative analysis of 3D motion from ultrasound images can provide improved information in several applications, such as arterial mechanical assessment, heart motion and blood flow. Unfortunately, it remains difficult to obtain a high definition of the motion estimate in the lateral and elevation directions (i.e. perpendicular to the beam axis). To increase the definition in both these directions, this paper presents a 3D extension of a the transverse oscillations method that enables one to obtain ultrasound fields featuring oscillations along the 3 spatial dimensions, using a single apodization function. The 3D motion method is estimated using the phases of the images. Simulation results show that a 3D trajectory can be followed with a relative mean error smaller than $8 \%$.
\end{abstract}

\section{INTRODUCTION}

Motion estimation is an essential part of ultrasound image sequence analysis. It can be used in various applications such as for example for speckle suppression [1], motion based image segmentation [2] and elastography [3]...

Several methods for two-dimensional ultrasound tissue motion tracking [4] and blood velocity estimation [5] have been proposed and clinically applied. Unfortunately, most of these methods cannot be applied to estimate the complete $3 \mathrm{D}$ motion or velocity vectors. With the emergence of ultrasound volumetric imaging, the need for a method that is capable of obtaining the complete $3 \mathrm{D}$ motion vector is even more pressing. Several ultrasound methods have been proposed for estimating 3D motion over the past decades. These include non-rigid image registration methods [6], or different speckle tracking methods [7-8]

Despite significant algorithmic advances, 3D motion estimation remains challenging due to limited field of view, low image quality, and large computation load.

To increase the definition of the motion estimation in the lateral and elevation directions (i.e. perpendicular to the beam axis), the transverse oscillations (TO) method has been recently extended in $3 \mathrm{D}$ by the group of J.A. Jensen [9]. This method enables one to obtain 2 separate ultrasound fields featuring each one oscillations along the axial dimension plus one perpendicular (lateral or elevation) direction. This approach consists more in a twice 2D tactic than 3D. In this approach, two different apodization functions are used for generating elevation and lateral oscillations, respectively.

This paper presents a method able to create transverse oscillations images (renamed here US-tagged images) featuring 3D oscillations using only one single apodization function, and a 3D motion estimation method based on the phases of such images. The paper first presents the 3D TO image formation method in the next section and describes the 3D phase-based motion estimation (PBME) method in Section III. Section IV presents the simulated 3D TO fields and the validation of the PBME on a series of $3 \mathrm{D}$ displacements of a simulated medium. The conclusion is given in Section V.

\section{THE 3D TRANSVERSE OSCILLATIONS METHOD}

\section{A. Introduction}

The 3D TO method is an extension of the 2D Transverse Oscillations approach [10-11]. Our study mainly focused on the apodization function and its incidence onto the expected point spread function (PSF). Hence, this section briefly refers to the $2 \mathrm{D}$ methodology in linear and sectorial approach, which easily allows the extension to 3D TO.

\section{B. 2D Apodization Function for Linear Scanning}

In conventional beamforming, the PSF shows oscillations only in the axial direction. On the other hand, in TO imaging, the lateral profile of the PSF, represented in Fig. 1a, can be expressed in eq. (1), which is a multiplication of a Gaussian envelope with a cosine function.

$$
h(x)=\cos \left(2 \pi x / \lambda_{x}\right) e^{-\pi\left(\frac{x}{\sigma_{x}}\right)^{2}}
$$

where $\lambda_{x}$ is the lateral wavelength and $\sigma_{x}$ is the half maximum width of the Gaussian envelop.

The beamforming is classically based on Fraunhofer approximation, the conditions of which are met at the focal point. In order to be able to apply the Fraunhofer approximation along the whole propagation path, the delay function is set so as to perform dynamic quadratic focusing. Under this approximation, the transverse profile of the PSF at the depth of focusing is related to the apodization function by a Fourier transform [12].

As a consequence the apodization function leading to the PSF profile given in eq 1 can easily be calculated. It is given in following eq 2 and represented in Fig. $1 b$.

$$
w_{r}(x)=\frac{1}{2}\left(e^{-\pi\left(\frac{x-x_{0}}{\sigma_{0}}\right)^{2}}+e^{-\pi\left(\frac{x+x_{0}}{\sigma_{0}}\right)^{2}}\right)
$$

where $x_{0}=\lambda z / \lambda_{x}$ and $\sigma_{0}=\sqrt{2} \lambda z / \sigma_{x}, \lambda$ is the wavelength of the transmitted pulsed, $z$ is the depth of interest, $\pm x_{0}$ is the position of the two peaks and $\pm \sigma_{0}$ is the half maximum width of each peak. 


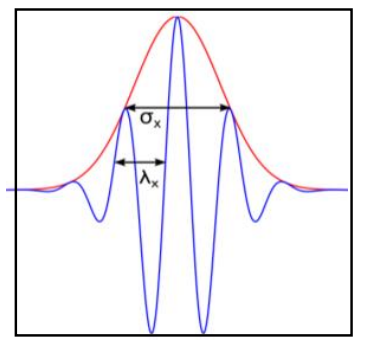

(a)

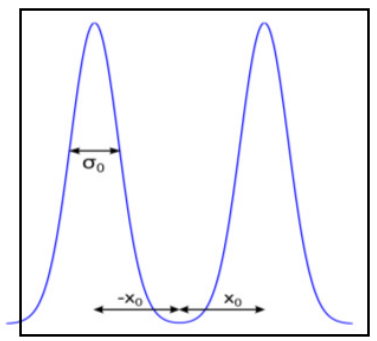

(b)
Figure 1: Representation of the lateral PSF profile (a) and the corresponding apodization function (b)

\section{2D Apodization Function for Sectorial Scanning}

The framework introduced above can be easily adapted to a conventional sectorial scanning. Indeed, the transformation of the model parameters from cartesian coordinates to polar coordinates is simple. This is done by setting $\lambda_{x}=\lambda_{\vartheta} r, \sigma_{x}=\sigma_{\vartheta} r$, and $z=r$, where $\lambda_{\vartheta}$ is the expected lateral wavelength of PSF profile in radians, $\sigma_{\vartheta}$ is the width at half maximum of PSF profile, and $r$ is the sweeping radius. Based on the approximation settings above, the position of two peaks $\pm x_{\vartheta_{0}}$ and the standard deviation $\sigma_{\vartheta_{0}}$ of the apodization function are given as follows:

$$
\left\{\begin{array}{l}
x_{\vartheta_{0}}=\lambda / \lambda_{\vartheta} \\
\sigma_{\vartheta_{0}}=\sqrt{2} \cdot \lambda / \sigma_{\vartheta}
\end{array}\right.
$$

Eq. (3) shows that $x_{\vartheta_{0}}$ and $\sigma_{\vartheta_{0}}$ are independent from the depth, but only limited by the values of the lateral wavelength and the half maximum width of the Gaussian envelop in radian. In other words, in the case of sectorial scanning the transverse oscillations lateral wavelength is no longer dependent on the depth which is a strong difference with linear scanning.

\section{3D Apodization Function for Pyramidal Scanning}

The 3D TO method requires a 2D transducer. In order to have a large field of view, two sectorial scanning, one for each transverse direction i.e. pyramidal scanning, is performed into the medium.

Instead of designing the 3D TO fields as Pihl, creating some oscillations in the axial and one transverse directions at at time (first lateral and then elevation), the 3D TO fields are built in order to oscillate in the axial and in the two transverse directions simultaneously. The apodization function allowing obtaining this specific field is an extrapolation of the apodization function presented in II.C. In the same way as one sectorial scanning is applied for each transverse direction, the previous apodization function is used in both directions, as follow:

$$
\begin{aligned}
& w_{r x}(x)=\frac{1}{2}\left(e^{-\pi\left(\frac{x-x_{0}}{\sigma_{0}}\right)^{2}}+e^{-\pi\left(\frac{x+x_{0}}{\sigma_{0}}\right)^{2}}\right), \text { with } x \in \mathbb{R}^{n} \\
& w_{r y}(y)=\frac{1}{2}\left(e^{-\pi\left(\frac{y-x_{0}}{\sigma_{0}}\right)^{2}}+e^{-\pi\left(\frac{y+x_{0}}{\sigma_{0}}\right)^{2}}\right), \text { with } y \in \mathbb{R}^{m} \\
& w_{r 2 D}(x, y)=w_{r x}(x) \times w_{r y}(y)^{\prime}, \text { with } w_{r 2 D} \in \mathbb{R}^{n \times m}
\end{aligned}
$$

Where $n$ and $m$ are the transducer dimensions, $\times$ is a matrical multiplication

These 3D images were generated by transmitting a plane wave and receiving with dynamic focalization and the aforementioned apodization function. The 2D apodization functions are illustrated in Fig. 2.

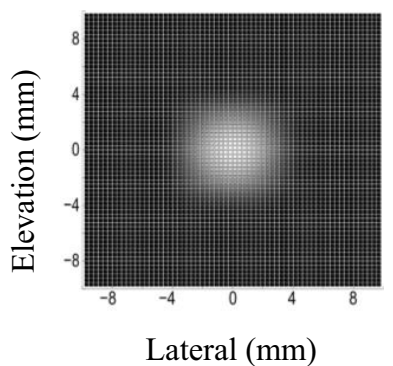

(a)

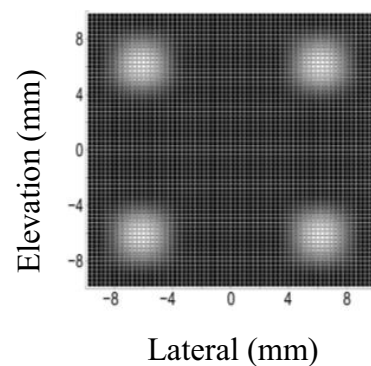

(b)
Figure 2: Apodization functions in transmit (a) and receive (b). Each small square represents an element of the matrix array. 256 active channels are used in this example. Colorbar: Black $\rightarrow$ white $=0->1$.

\section{THE 3D PHASE-BASED MOTION ESTIMATION METHOD}

\section{A. Algorithm Description}

In this section, the description of the 3D phase-based block matching method is proposed. This technique is the 3D extension of the 2D method proposed by Basarab et al [13]. A pair of 3D RF volumes of interest with $\mathrm{TO}, i_{1}(x, y, z)$ and $i_{2}(x, y, z)$ representing the same medium before and after the application of a displacement, are considered. The relation between the 2 volumes is:

$$
i_{1}(x, y, z)=i_{2}\left(x+d_{x}, y+d_{y}, z+d_{z}\right)
$$

where $d_{x}, d_{y}$, and $d_{z}$ are the spatial displacements to be estimated in each pixel $(x, y, z)$.

\section{B. Phase of Analytical Signal}

In contrast with classical block matching, the proposed method uses 6 phase images instead of amplitude images $i_{1}$ and $i_{2}$. 3D phase images are obtained using 3 single octants analytical signals applied to $i_{1}$ and $i_{2}$ and defined in the Fourier domain. Usually, for each of the 2 images, 8 single octants analytical signals can be defined, 


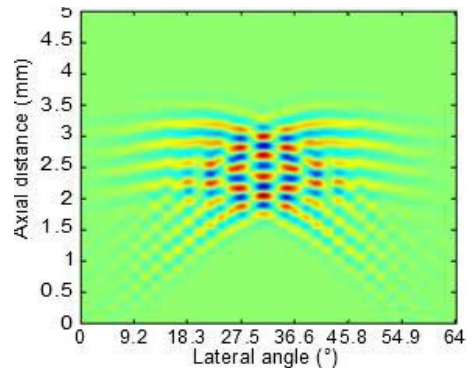

(a)

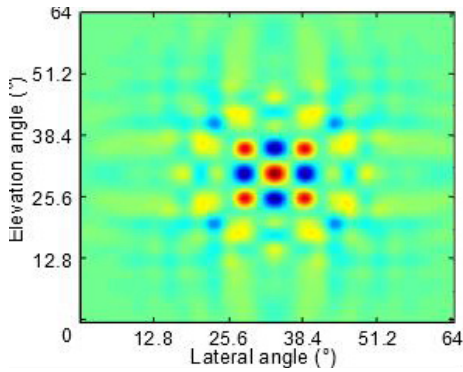

(b)

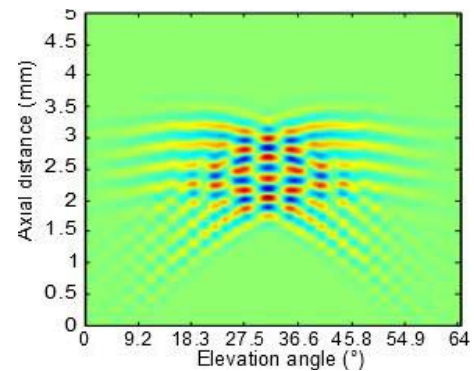

(c)

Figure $3: 3 \mathrm{D}$ PSF at $40 \mathrm{~mm}$ of depth with TO in both (lateral and elevation) directions at the same time. (a)Axial/Lateral view, (b) Elevation/Lateral view, (c) Axial/Elevation view.

and correspond to each octant of the Fourier spectrum. Here, only 3 of such analytical signals are considered. These 6 complex signals, noted $i_{11}, i_{12}, i_{13}, i_{21}, i_{22}$, and $i_{23}$, are defined in the Fourier domain as shown in eq. (8).

$$
\begin{aligned}
& I_{11}=I_{1} \cdot\left(1+\operatorname{sgn}\left(f_{x}\right)\right)\left(1+\operatorname{sgn}\left(f_{y}\right)\right)\left(1+\operatorname{sgn}\left(f_{z}\right)\right) \\
& I_{12}=I_{1} \cdot\left(1+\operatorname{sgn}\left(f_{x}\right)\right)\left(1-\operatorname{sgn}\left(f_{y}\right)\right)\left(1+\operatorname{sgn}\left(f_{z}\right)\right) \\
& I_{13}=I_{1} \cdot\left(1+\operatorname{sgn}\left(f_{x}\right)\right)\left(1-\operatorname{sgn}\left(f_{y}\right)\right)\left(1-\operatorname{sgn}\left(f_{z}\right)\right) \\
& I_{21}=I_{2} \cdot\left(1+\operatorname{sgn}\left(f_{x}\right)\right)\left(1+\operatorname{sgn}\left(f_{y}\right)\right)\left(1+\operatorname{sgn}\left(f_{z}\right)\right) \\
& I_{22}=I_{2} \cdot\left(1+\operatorname{sgn}\left(f_{x}\right)\right)\left(1-\operatorname{sgn}\left(f_{y}\right)\right)\left(1+\operatorname{sgn}\left(f_{z}\right)\right) \\
& I_{23}=I_{2} \cdot\left(1+\operatorname{sgn}\left(f_{x}\right)\right)\left(1-\operatorname{sgn}\left(f_{y}\right)\right)\left(1-\operatorname{sgn}\left(f_{z}\right)\right)
\end{aligned}
$$

Where $I_{1}$ and $I_{2}$ are the 3D Fourier transforms of the ultrasound volumes $i_{1}$ and $i_{2}$. The phase images are then extracted from these 6 analytical signals, and denoted $\phi_{11}$, $\phi_{12}, \phi_{13}, \phi_{21}, \phi_{22}$ and $\phi_{23}$, respectively.

\section{Motion Estimation}

As explained by Basarab et al [14], if the 3D signals extracted from volumes $i_{1}$ and $i_{2}$ follow the model in (10), the mean value of phase differences between the same type of complex signals corresponding to signal $i_{1}$ and $i_{2}(11)$, is linked to the displacement between both volumes of interest(12).

$$
\begin{aligned}
& \left\{\begin{aligned}
s_{1}(x, y, z) & =\omega_{s 1}(x, y, z) \cos \left(2 \pi f_{x}\left(x-d_{x 1}\right)\right) \\
& \times \cos \left(2 \pi f_{y}\left(y-d_{y 1}\right)\right) \cos \left(2 \pi f_{z}\left(z-d_{z 1}\right)\right) \\
s_{2}(x, y, z) & =\omega_{s 2}(x, y, z) \cos \left(2 \pi f_{x}\left(x-d_{x 2}\right)\right) \\
& \times \cos \left(2 \pi f_{y}\left(y-d_{y 2}\right)\right) \cos \left(2 \pi f_{z}\left(z-d_{z 2}\right)\right)
\end{aligned}\right. \\
& \left\{\begin{array}{l}
\phi_{1}(x, y, z)=\phi_{11}(x, y, z)-\phi_{21}(x, y, z) \\
\phi_{2}(x, y, z)=\phi_{12}(x, y, z)-\phi_{22}(x, y, z), \\
\phi_{3}(x, y, z)=\phi_{13}(x, y, z)-\phi_{23}(x, y, z)
\end{array}\right. \\
& \hat{d}_{x}=\frac{\bar{\phi}_{1}-\bar{\phi}_{2}}{4 \pi f_{x}} ; \hat{d}_{y}=\frac{\bar{\phi}_{2}-\bar{\phi}_{3}}{4 \pi f_{y}} ; \hat{d}_{z}=\frac{\bar{\phi}_{3}+\bar{\phi}_{1}}{4 \pi f_{z}}
\end{aligned}
$$

Where $\omega_{\mathrm{s}}$ are some windows modulating a little the shape of the oscillations., $d_{x}=d_{x 1}-d_{x 2}, d_{y}=d_{y 1}-d_{y 2}$, and $d_{z}=d_{z 1}-d_{z 2}$

Note that the PBME estimator has several advantages, but it cannot estimate a displacement larger than half a wavelength of the oscillations in each direction.

\section{RESTULTS}

\section{A. Simulation Description}

The simulations are performed using the ultrasound simulation program Field II [15]. All results are obtained with the same $2 \mathrm{D}$ phased array and simulation parameters are shown in Table I.

The beamforming parameters has been calculated in order to have a transverse spatial frequency of $3.7 \mathrm{rad}^{1}$ under the Gaussian envelop. First, the 3D TO method is verified with a simulated point spread function (PSF), then the PBME is validated on a simulated 3D displacement.

Table I. Parameter setting for the simulation

\begin{tabular}{ll}
\hline \hline Parameter & Value \\
\hline Transducer & \\
Transducer & $2 \mathrm{D} \mathrm{Phased} \mathrm{array}$ \\
Number of elements & $64 \times 64$ \\
Center frequency & $3 \mathrm{Mhz}$ \\
Pitch & $\lambda / 2$ \\
Kerf & $\lambda / 100$ \\
\hline Setup parameters & \\
Sampling Frequency & $50 \mathrm{Mhz}$ \\
Speed of sound & $1540 \mathrm{~m} / \mathrm{s}$ \\
No. of transmit cyles & 3 \\
Transmit focus & Plane wave \\
Receive focus & $5,10,15 \ldots 40 \mathrm{~mm}$ \\
Transmit apodization & $2 \mathrm{D} \mathrm{Hanning} \mathrm{windows}$ \\
Receive apodization & 4 distant peaks \\
Number of lines & $128 \times 128$ \\
Volume width & $45^{\circ} \times 45^{\circ}$ \\
\hline
\end{tabular}

\section{B. 3D Beamgorming Validation}

To investigate how well defined the 3D transverse oscillations are, one PSF is calculated with Field II. The 3D PSF obtained with the specified apodizations of eq (6) is illustrated in Fig. 3. The theoretical lateral spatial wavelength is $0.08 \mathrm{rad}^{-1}$, whereas the mean wavelength is $0.074 \mathrm{rad}^{-1}$. This gives a bias of $7.5 \%$ between the theoretical and the mean values of the transverse spatial frequencies.

\section{PBME Method Validation}

In order to validate the proposed method, the PBEM method is first validated on a single transverse motion. A random reflector map was generated and translated with a pure lateral displacement. A displacement in the range $[0$; 1.5] $\mathrm{mm}$ with a $0.1 \mathrm{~mm}$ increments was used. A grid of $20 \times 10 \times 10$ points in the volume of interest was used at a depth of $30 \mathrm{~mm}$ i.e. which corresponded to a volume of $0.3 \times 3.7 \times 3.7 \mathrm{~mm}^{3}(\mathrm{z}, \mathrm{x}, \mathrm{y})$. 
The resulting estimations of the displacement are presented in Fig. 4

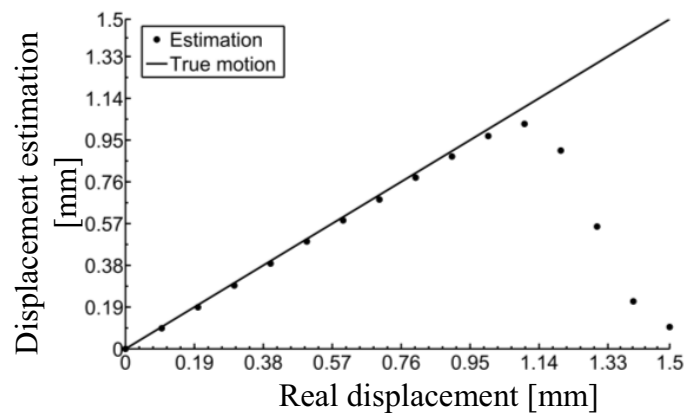

Figure. 4: Motion estimation results. The PBME estimation correctly follows the real trajectory until a displacement equal to $1.05 \mathrm{~mm}$. corresponding to the mid-wavelength.

The motion is correctly measured by the PBME method with a mean relative error of $0.5 \%$ until a displacement of $1.05 \mathrm{~mm}$. The spatial wavelength is equal to $2.22 \mathrm{~mm}$ at a depth of $30 \mathrm{~mm}$, hence the half wavelength is $1.11 \mathrm{~mm}$. The theory claiming that the maximum displacement that can be estimated corresponds to the half wavelength is perfectly verified.

In order to evaluate the PBEM method, a 3D trajectory situation is also investigated. Using the same way, a $3 \mathrm{D}$ trajectory is simulated by Field II. The axial, lateral and elevation maximum displacement amplitudes of displacement are $0.25,1$ and $1 \mathrm{~mm}$, respectively. The PBME method is evaluated on 10 different simulations. The applied motion corresponds to an elliptical trajectory. The results is shown in Fig.5

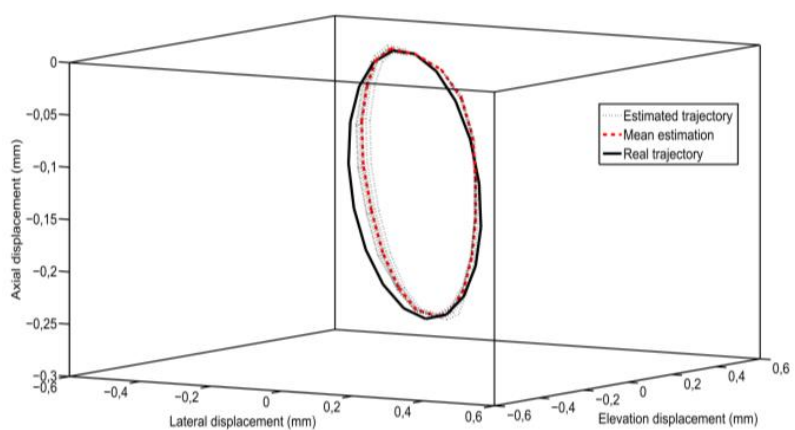

Figure 5: 3D trajectory estimated by the proposed PBME method.

The estimated trajectory follows the real trajectory with a maximum error of $3 \mu \mathrm{m}$ in axial, $70 \mu \mathrm{m}$ in lateral, and 72 $\mu \mathrm{m}$ in elevation. In $\mu \mathrm{m}$, the error (mean \pm standard deviation) is $2 \pm 1,40 \pm 30$, and $41 \pm 31$ in axial, lateral andelevation, respectively. The mean relative error $5 \pm 1,6$ \pm 1 , and $6 \pm 2$ in axial, lateral and elevation directions, respectively.

\section{CONCLUSION}

In this paper, we have first presented a new 3D TO beamforming method able to synthesize oscillations simultaneously in the three spatial directions.

Second, a PBME methodology exploiting such 3D TO imaging has been investigated. All three spatial displacements are estimated simultaneous by the presented method and are assessed with a mean relative error inferior at $8 \%$.

\section{ACKNOWLEDGMENT}

This work was performed within the framework of the LABEX CELYA (ANR-10-LABX-0060) PRIMES (ANR11-LABX-0063) of Universite de Lyon, within the program "Investissements d'Avenir" (ANR-11-IDEX-0007) operated by the French National Research Agency (ANR).

\section{REFERENCE}

[1].Cheng-Hsien Lin, Yung-Nien Sun, Chii-Jeng Lin. "A Motion Compounding Technique for Speckle Reduction in Ultrasound Images" Journal of Digital Imaging June 2010, Volume 23, Issue 3, pp 246-257

[2] T. Dietenbeck, D. Barbosa, M. Alessandrini, R. Jasaityte, V. Rosebyn, J. D'hooge, O. Bernard, and D. Friboulet, "Multiview myocardial tracking in echocardiographic 2D sequences using shape and motion constrained level-set", IEEE International Symposium on Biomedical Imaging (ISBI), San Fransisco, California, USA, pp. 1010-1013, 2013.

[3] E. Brusseau, J. Fromageau, N. Rognin, P. Delachartre, and D. Vray, "Investigating elastic properties of soft biological tissues", IEEE Eng Med Biol, vol. 21, no. 4, pp. 86-94, 200

[4] Zahnd G, Boussel L, Marion A, Durand M, Moulin P, Sérusclat A, Vray D. "Measurement of two-dimensional movement parameters of the carotid artery wall for early detection of arteriosclerosis: a preliminary clinical study." Ultrasound in Medecine \& Biology, vol. 37, no. 9, pp. 1421-1429, 2011

[5] Erik Gudmundson, Andreas Jakobsson, Jørgen A. Jensen, Petre Stoica, "Blood velocity estimation using ultrasound and spectral iterative adaptive approaches", Signal Processing, Volume 91, Issue 5, May 2011, Pages 1275-1283, ISSN 0165-1684.

[6]. Elen, A., Choi, H.F., Loeckx, D., Gao, H., Claus, P., Suetens, P., Maes, F., D'hooge,J.: "Three-dimensional cardiac strain estimation using spatiotemporal elastic registration of ultrasound images: A feasibility study." TMI 27(11) (2008) 1580\{1591

[7].Chen, X., Xie, H., Erkamp, R., Kim, K., Jia, C., Rubin, J.M., O'Donnell, M. "3-D correlation-based speckle tracking”. Ultrasonic Imaging 27, 21-36 (2005)

[8] L. N. Bohs, B. J. Geiman, M. E. Anderson, S. C. Gebhart, andG. E. Trahey, "Speckle tracking for multi-dimensional flow estimation," Ultrasonics, vol. 38, pp. 369-375, 2000.

[9] M. J. Pihl and J. A. Jensen, "3D velocity estimation using a 2D phased array," in Proc. IEEE Ultrason. Symp, 2011, pp. 430-433.

[10] Jensen J. A., Munk, P., "A new method for estimation of velocity vectors", IEEE Trans Ultrasonics Ferroelectrics , and Frequency Control, vol. 45, no. 3, pp. 837-851, 1998.

[11] H. Liebgott, A. Basarab, P. Gueth, D. Friboulet, P. Delachartre "Transverse oscillations for tissue motion ,estimation" Ultrasonics, vol. 50, no. 6 , pp. $548-555,2001$

[12] J. W. Goodman, Introduction to Fourier optics: Roberts \& Company Publishers, 2005.

[13] A. Basarab, H. Liebgott, and P. Delachartre, "Analytic estimation of subsample spatial shift using the phases of multidimensional analytic signals", IEEE Trans Image Proces, vol. 18, no. 2, pp. 440-447, 2009

[14] A. Basarab, P. Gueth, H. Liebgott, and P. Delachartre, "Phase-based block matching applied to motion estimation with unconventional beamforming strategies", IEEE Trans Ultrason Ferroelectr Freq Control, vol. 56, no. 5, pp. 945-957, 2009

[15] J. A. Jensen, "Field: A Program for Simulating Ultrasound Systems," Med. Biol. Eng. Comp., vol. 10th Nordic-Baltic Conference on Biomedical Imaging, Vol. 4, Supplement 1, Part 1, pp. 351-353, 1996. 